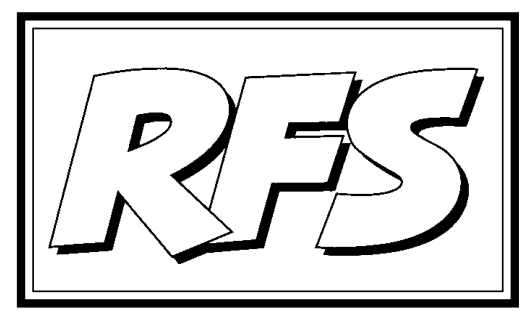

Revista de Fomento Social, 56 (2001), 211-232

\title{
Las claves para valorar la nueva financiación autonómica ${ }^{1}$
}

\section{Araceli DE LOS RÍOS BERJ ILLOS²}

\section{Introducción}

En julio del presente año 2001, el gobierno central acordó con los gobiernos autonómicos, el próximo modelo de financiación de las CCAA. Lo que se ha aprobado es cuáles van a ser los ingresos con que van a contar las CCAA para financiar las competencias transferidas, y de dónde van a proceder dichos recursos. Esta es, sin duda, una de las cuestiones más importantes del Estado de las Autonomías; también lo son el reparto del poder político y de las competencias económicas, pero téngase en cuenta que, ni la actividad política, ni la económica, pueden llevarse a cabo sin los recursos financieros necesarios.

1 Este artículo recoge algunas de las principales aportaciones de la tesis doctoral de la autora, titulada Una propuesta de financiación para las Comunidades Autónomas españolas de régimen común. Un enfoque económico, dirigida por el Dr. D. Adolfo Rodero Franganillo, cuya experiencia y dedicación la hicieron posible.

2 Profesora del Departamento de Gestión Empresarial y Métodos Cuantitativos de la Facultad de CC.EE. y Empresariales (ETEA) adscrita a la Universidad de Córdoba. 
Este artículo tiene dos objetivos principales, por una parte, pretendemos ofrecer al lector las claves para valorar las virtudes y los defectos de un modelo de financiación regional, dichas claves vienen dadas por los principios que deben inspirar el sistema de financiación autonómica; por otra parte, realizamos una valoración del modelo aprobado por el gobierno identificando sus principales aportaciones e insuficiencias.

Una cuestión previa que hay que tener presente es que la financiación autonómica no tiene una solución óptima, por varias razones; la primera es que al tratarse de un problema económico (de asignación de recursos escasos a fines alternativos), la solución dependerá de los valores o principios que los distintos agentes decisores (en este caso políticos) apliquen en su actuación; la segunda, es que las realidades socioeconómicas de las regiones españolas son distintas, por lo que encontrar un sistema que satisfaga por igual a todas las CCAA es prácticamente imposible; así por ejemplo, mientras que las CCAA más desarrolladas demandan una mayor autonomía financiera, las CCAA menos desarrolladas reclaman que los avances en la autonomía se logren sin poner en peligro la solidaridad del sistema.

La solución adoptada dependerá del consenso al que lleguen las distintas administraciones autonómicas con la administración central, que a su vez va a depender de los principios que apliquen en su actuación. Esta cuestión, los principios que deben inspirar el sistema de financiación regional, es clave a la hora de proponer un modelo de financiación autonómica. De hecho, cualquier modelo es el resultado de una determinada combinación de los mismos. Ocupémonos por tanto de cuáles son estos principios, quéobjetivos pueden alcanzarse a través los mismos, y qué repercusiones económicas se derivan de ellos. Tener claras estas cuestiones nos permitirá valorar cualquier actuación política en este ámbito.

\section{Los principios de la financiación autonómica}

Analizar los principios del sistema de financiación autonómica, equivale a examinar cuáles son las virtudes que debe tener un modelo de financiación regional. En este sentido, el sistema debe ser tal que dote a las CCAA de los recursos suficientes para prestar las competencias transferidas, que otorgue a las CCAA la autonomía necesaria para desarrollar dichas competencias, pero que al mismo tiempo contemple la solidaridad entre las regiones 
españolas, y la necesaria coordinación con la actuación de la administración central. Por tanto, los principios a los que nos referimos son: suficiencia, autonomía, solidaridad y coordinación. Lo que tratamos que explicar a continuación es qué es la suficiencia, la autonomía, la solidaridad, la coordinación, para qué sirven estos principios, y qué ocurre si el sistema carece de suficiencia, autonomía, solidaridad y coordinación, así como en qué recursos financieros se traduce cada uno de estos principios en un modelo de financiación.

Conceptos, objetivos e implicaciones económico-financieras:

\section{a) Suficiencia}

La suficiencia implica que el sistema de financiación dote a las CCAA de los recursos necesarios para la prestación de los servicios públicos transferidos.

El objetivo del principio de suficiencia es, por tanto, dotar a las CCAA de los recursos necesarios para financiar el gasto correspondiente a las competencias transferidas.

La suficiencia financiera contribuye al equilibrio presupuestario de las CCAA, y al logro de los objetivos propios del principio de autonomía financiera.

\section{b) Autonomía financiera}

La autonomía financiera es la capacidad de una comunidad para decidir la composición y el nivel de prestación de los servicios públicos que le han sido transferidos, y de fijar el volumen y la composición de los ingresos. En definitiva, que los gobiernos autonómicos sean responsables del gasto, pero también de sus ingresos.

Los objetivos de la autonomía financiera pueden agruparse en tres ámbitos: político, hacendístico y económico-financiero. Respecto al primero, la autonomía financiera dota de efectividad a la autonomía política. En el ámbito de la Hacienda Pública, permite a las CCAA configurar una hacienda propia, reduciendo su dependencia de las transferencias del Estado. En el aspecto económico-financiero, la autonomía dota a las comunidades de la 
capacidad financiera para mejorar el nivel de prestación de los servicios públicos según las preferencias de los ciudadanos, y por otra parte, hace posible el aumento de la eficiencia en la prestación del gasto público.

La presencia de autonomía financiera en el sistema puede dar lugar a mejoras en el nivel de prestación de los servicios públicos, así como a una asignación más eficiente del gasto público en general. Además, puede contribuir a un mayor equilibrio presupuestario, en la medida en que las CCAA pueden ejercer su capacidad normativo-tributaria para financiar los aumentos del gasto, en vez de recurrir al endeudamiento. La ausencia de autonomía financiera daría lugar a efectos contrarios a los indicados.

\section{c) Solidaridad}

La solidaridad es la redistribución de recursos que se realiza con la finalidad de equiparar los niveles de progreso de todas las regiones.

Los principales objetivos del principio de solidaridad son dos: establecer un equilibrio económico adecuado y justo entre las diversas partes del territorio nacional, y alcanzar la equidad. El primero se concreta en la reducción de las desigualdades económicas interterritoriales, y el segundo en la nivelación de los servicios públicos, garantizando la igualdad de acceso a todos los ciudadanos.

La articulación del principio de solidaridad en el sistema de financiación autonómica contribuirá a que se reduzcan las desigualdades económicas entre las regiones. No obstante, el logro de este objetivo no se puede atribuir exclusivamente a los mecanismos recogidos en el sistema de financiación autonómica, ya que para su realización requiere del conjunto de las políticas económicas del Estado. Sin embargo, la ausencia de solidaridad en el sistema contribuiría a que incrementasen las desigualdades interterritoriales, principalmente porque se limitarían las posibilidades de crecimiento de las regiones más desfavorecidas.

\section{d) Coordinación}

La coordinación implica que los distintos niveles de las Administración Pública se pongan de acuerdo en su actuación con la finalidad de incrementar la eficiencia de las funciones asignadas a cada nivel de gobierno. 
El objetivo perseguido por el principio de coordinación es lograr una mayor eficiencia de las principales políticas económicas en las que comparten competencias los distintos niveles de la Administración Pública, por ejemplo, la política de inversiones públicas, o la de distribución del gasto público.

En materia de política económica la coordinación contribuye al logro de los objetivos de la política de estabilización, en concreto ha permitido la reducción de los niveles de déficit y endeudamiento autonómico, y por otra parte ha contribuido a la convergencia real de las regiones españolas con las europeas, en la medida en que se ha producido una concentración de las inversiones en las regiones menos desarrolladas. En materia presupuestaria la coordinación repercute mejorando la gestión de los ingresos y gastos públicos.

Concreción de los principios en el sistema de financiación

En virtud del principio de autonomía, una parte de los recursos de las CCAA deben proceder de ingresos de carácter tributario, que pueden ser propios o cedidos total o parcialmente por el Estado. Para que exista una auténtica autonomía, los gobiernos autonómicos no deben sólo apropiarse del rendimiento de las figuras tributarias, sino quetambién deben contar con capacidad normativa propia o cedida sobre las mismas. La capacidad normativo-tributaria propia se refiere a que las CCAA pueden crear sus propios tributos o establecer recargos sobre los tributos del Estado, tal y como se recoge en la Ley Orgánica de Financiación de las CCAA; la capacidad normativo-tributaria cedida se refiere a que el Estado cede a las CCAA la posibilidad de modificar algún o algunos parámetros de un tributo estatal cedido, con la finalidad de alterar la recaudación obtenida del mismo; todo ello dentro de los márgenes establecidos en la Ley de Cesión de Tributos.

En cambio, el principio de solidaridad se instrumenta a través de las transferencias. En teoría pueden existir flujos financieros entre CCAA, que se articularían mediante un fondo de suma cero (transferencias horizontales), y transferencias de recursos de la Administración central a las CCAA (transferencias verticales). En el sistema de financiación de las comunidades autónomas los recursos que hacen efectivo el principio de solidaridad son estos últimos.

Hasta 1997, los ingresos autonómicos procedían en su mayor parte de 
transferencias del Estado, sin embargo, existía un amplio consenso sobre la necesidad de reducir la financiación vía transferencias, y aumentar la autonomía del sistema mediante la cesión de nuevos tributos a las CCAA. Esta situación, que marcó la tendencia de la financiación autonómica hasta la actualidad, nos lleva a plantearnos las siguientes cuestiones: ¿por qué no es deseable que las CCAA se financien mayoritariamente con transferencias del Estado?, y por otra parte, ¿por qué son necesarias dichas transferencias?

Respecto a la primera cuestión, hay que indicar que la justificación económica del propio proceso de descentralización es que la prestación del gasto público por los niveles de gobierno más próximos al ciudadano es más eficiente. La eficiencia también está directamente relacionada con la percepción por parte de los ciudadanos de los beneficios y costes que se derivan de dicho gasto público.

Cuando las competencias traspasadas a los gobiernos regionales se financian con transferencias estatales, se generan efectos de ilusión fiscal, es decir, el ciudadano no relaciona el beneficio obtenido con el coste de su financiación; además, los gobiernos regionales al no ser responsables de los ingresos, siempre pueden trasladar la responsabilidad sobre la insuficiencia de recursos o el deficiente nivel de prestación de los servicios al gobierno central. Todo ello podría dar lugar a una asignación del gasto no eficiente, y por estas razones la autonomía y la corresponsabilidad fiscal deben presidir el sistema de financiación de las comunidades autónomas.

Respecto a la segunda pregunta, las transferencias son necesarias para compensar las siguientes situaciones:

a) Los desequilibrios fiscales horizontales, es decir, las diferencias entre los gobiernos regionales para obtener recursos fiscales, dichas diferencias determinan que para un mismo esfuerzo fiscal la provisión de bienes y servicios sea distinta.

b) Los desequilibrios fiscales verticales, es decir, las diferencias entre la capacidad fiscal de cada región y sus necesidades de financiación o gasto; existen factores socioeconómicos que justifican las diferencias en el coste de prestación de los servicios públicos, por ejemplo, la dispersión de la población.

c) La presencia de externalidades en la prestación de los servicios públicos. El hecho de que los beneficiarios del gasto no sean sólo los residentes de una determinada región, justifica que el gobierno central 
transfiera recursos para su financiación.

Además, en el caso concreto de las CCAA, la dotación inicial de capital público difiere de una regiones a otras, así como el nivel de prestación de los servicios públicos. Dado que estas insuficiencias las heredan las CCAA, desde nuestro punto de vista ha de ser el gobierno central el que las compense mediante transferencias complementarias de los recursos con que se financien las competencias transferidas.

\section{El equilibrio entre los principios financieros}

En un modelo de financiación regional hay que determinar cuánta autonomía es necesaria, cuánta solidaridad, cuánta coordinación, y cuánta suficiencia. La respuesta a estas preguntas es una decisión política, por tanto nuestro objetivo es poner de manifiesto aquellos aspectos que hay que tener presentes. Sobre ello hay tres cuestiones fundamentales: 1) los límites constitucionales a la aplicación de dichos principios, es decir, el papel que la Constitución otorga a cada principio, 2) las interrelaciones entre los mismos, y 3) las circunstancias en que se desarrolló el proceso de descentralización pública en España.

\section{1) Los principios en la Constitución}

La Constitución no otorga un papel predominante a ninguno de los principios, en concreto, al referirse al principio de autonomía establece que, la autonomía se ejercerá con arreglo a los principios de coordinación y solidaridad. Una primer idea es, por tanto, que constitucionalmente ningún principio es más importante que el resto. Esto a su vez nos lleva a una segunda afirmación, la aplicación de los principios tiene como límites generales que:

a) Cada uno se aplicará para el logro de los fines que le son propios, de lo que se deduce que en virtud del principio de autonomía, no cabe reclamar una apropiación ilimitada de los recursos correspondientes a la capacidad financiera de la región.

b) Cada principio debe aplicarse de tal forma que no impida el logro de los objetivos propios de los demás. Es decir, ni la solidaridad, ni la coordinación pueden dejar sin contenido la autonomía de las regiones, ni viceversa. 


\section{2) Relaciones entre los principios}

El principio de autonomía puede provocar tensiones en el principio de equidad que deben ser compensadas por la Administración central en virtud de la solidaridad. Una mayor autonomía puede dar lugar a desigualdades financieras entre las regiones, porque, como ya se ha indicado, existen diferencias en la capacidad financiera de las comunidades, y en el coste de prestación de los servicios públicos, además el nivel de partida de cada comunidad fue diferente. La solidaridad es necesaria para evitar que estas diferencias se incrementen y perpetúen.

El principio de suficiencia está vinculado al de autonomía y al de solidaridad. La suficiencia es una condición necesaria aunque no suficiente para que exista autonomía. Por otra parte, en la instrumentación del principio de suficiencia se ha tenido en cuenta el distinto coste de prestación de los servicios públicos en cada región, de lo que cabe deducir que la cobertura del coste de los servicios es una cuestión de suficiencia pero también de solidaridad.

El principio de coordinación también está relacionado con los principios de autonomía y solidaridad, ya que la necesidad de coordinación aumenta al hacerlo el grado de autonomía; y el grado en que se alcancen los objetivos económicos propios del principio de solidaridad, también depende del grado en que se coordinen las políticas de redistribución einversión de las distintas Administraciones españolas y de la Unión Europea.

\section{3) El proceso de descentralización pública en España}

Respecto a esta tercera cuestión, tenemos que tener presente que las regiones españolas nacen del proceso de descentralización de un Estado único centralizado. Este proceso es distinto del que surgen los Estados federales, en el que son los gobiernos regionales o Estados quienes ceden parte de su poder a una gobierno superior o federal. Esta cuestión política no es independiente del problema económico que nos ocupa. Así, por ejemplo, mientras que las diferencias entre los Estados de un gobierno federal se toleran (en la medida en que se heredan de los Estados independientes originarios), a un Estado centralizado cabe pedirle que en el momento inicial de traspaso de competencias, el nivel de prestación de los servicios y la dotación inicial de capital fuera la misma en cada región. El hecho de que esto no fuera así, y que por tanto, el punto de partida de cada comunidad autónoma fuera diferente, justifica que en el caso español, la solidaridad sea

\section{RFS}


un objetivo tan importante como el de la autonomía de las regiones.

Como consecuencia de todo lo anterior, se concluye que en la financiación de las CCAA el principio de autonomía debe compartir protagonismo con los principios de solidaridad y coordinación, porque así lo establece la Constitución, y porque no se debe prescindir del contexto y el procedimiento en que se llevó a cabo la descentralización del sector público español. El principio que mejor define cómo debe ser el sistema de financiación de las CCAA es el de autonomía-solidaria.

\section{Una valoración del nuevo modelo de financiación autonómica}

Como se desprende del análisis anterior, los dos ejes principales del sistema de financiación regional son: la articulación del principio de autonomía y la del principio de solidaridad. La primera se concreta en consensuar una cesta de tributos como principal fuente de financiación de las CCAA, y la segunda en adoptar un criterio que dote de equidad al sistema, y según el cual se redistribuirán las transferencias de nivelación.

Aunque a la fecha de cierre del presente texto (julio 2001) aún no se conocen todas las particularidades del nuevo modelo de financiación autonómica, contamos con sus líneas generales, tanto en lo que se refiere a la articulación de la autonomía como de la solidaridad.

Una breve referencia a la metodología de los modelos anteriores

Antes de entrar a valorar el nuevo modelo, nos ha parecido interesante exponer cuál es el procedimiento según el cual se determina la financiación total correspondiente a cada comunidad autónoma.

En todos los modelos se parte de un volumen de financiación incondicionada $^{3}$ total correspondiente a las CCAA; en concreto, en el nuevo modelo que

3 Los recursos totales de los que disponen las CCAA pueden clasificarse en dos grandes partidas, recursos de carácter incondicionado, cuyo destino pueden fijar libremente los gobiernos autonómicos, y recursos de carácter condicionado, cuyo destino viene fijado por la administración central o una entidad supranacional, como por ejemplo, la Unión Europea. Los recursos correspondientes a la financiación de las competencias transferidas son en su mayoría recursos de carácter incondicionado; la exposición de la metodología empleada para determinar la financiación correspondiente a cada comunidad autónoma, se refiere a este tipo de recursos. 
entrará en vigor a partir del 1 de enero de 2002, este montante de recursos es de 8,57 billones de ptas., en 1997 fue de 3,05 billones de ptas. Este volumen de recursos se fija en función de las competencias transferidas a las CCAA; en este nuevo modelo el aumento de recurso es tan considerable porque ya se han transferido todas las competencias en educación a las CCAA que aún quedaban pendientes (también está previsto el traspaso de las competencias en materia sanitaria). Pero también hemos de tener presente que en todos los modelos negociados desde 1987 se partía de una restricción, y es que el nuevo modelo resultante de las negociaciones no podía aportar a ninguna comunidad autónoma menos recursos de los que venía percibiendo por el modelo que finalizaba. Podemos buscar una explicación financiera a esta restricción, pero fundamentalmente lo que facilitaba era el desarrollo de la negociación política; es evidente que el consenso se alcanza más fácilmente cuando no hay nada que perder.

Los mecanismos del sistema que van a aportar dicha financiación varían de un modelo a otro; hasta 1992 los más importantes eran la participación en los ingresos del Estado (PIE), los tributos cedidos y las tasas afectas a los servicios transferidos; en 1993 se incorpora una participación territorializada en el IRPF, y en 1997 ad emás de los anteriores recursos secede parcialmente el IRPF a las CCAA. Tomando un año como referencia (año base) se estima el valor de los tributos cedidos, tasas, participación territorializada en el IRPF y el rendimiento del IRPF cedido a las CCAA (es lo que se conoce como valores normativos).

A partir de las variables socioeconómicas y sus respectivas ponderaciones, se establecen unas fórmulas que permiten calcular el volumen de financiación incondicionada correspondiente a cada comunidad. Dichas variables eran: la población, la superficie, la dispersión, la insularidad y las unidades administrativas; además había dos variables redistributivas o de suma nula, la pobreza relativa y el esfuerzo fiscal. El primer grupo de variables se consideran indicadores del coste de prestación de los servicios en cada comunidad.

En el modelo de 1987 existió una fórmula para determinar la financiación de las competencias en educación (que inicialmente sólo se habían transferido a algunas (CAA), y otra para la financiación del resto de competencias que eran comunes a todas las CCAA. De tal forma que la financiación total correspondiente a una comunidad a la que se le habían traspasado las competencias comunes y las de educación, era la correspondiente a la suma 
del resultado de cada una de las dos fórmulas. En el modelo de 1992 esto cambia y en vez de los dos bloques de competencias (educación, y las competencias comunes), se determina directamente la financiación total de las comunidades autónomas del artículo 151 de la Constitución (CCAA a las que se le habían transferidos ambos grupos de competencias) y de las del 143 (CCAA a las que sólo se les había transferido las competencias comunes).

Una vez fijada, mediante la aplicación de estas fórmulas, la financiación correspondiente a cada comunidad autónoma, se procede a determinar la parte de dicha volumen de recursos que cada comunidad autónoma va a percibir por participación en los ingresos del Estado.

Hasta 1993 $\mathrm{PIE}_{\mathrm{i}}=\mathrm{FF}_{\mathrm{i}}-\mathrm{TC}_{\mathrm{i}}$

De 1994 a 1996 $\mathrm{PIE}_{\mathrm{i}}=\mathrm{FF}_{\mathrm{i}}-\mathrm{TC}_{\mathrm{i}}-15 \% \mathrm{IRPF}_{\mathrm{i}}$

De 1997 a 2001 $\mathrm{PIE}_{\mathrm{i}}=\mathrm{FF}_{\mathrm{i}}-\mathrm{TC}_{\mathrm{i}}-\mathrm{T}_{\text {Tarifa autonómica }}-15 \% \mathrm{IRPF}_{\mathrm{i}}$ Siendo:

$\mathrm{PIE}_{\mathrm{i}}$, la participación en los ingresos del Estado correspondiente a la comunidad autónoma i.

$\mathrm{FF}_{\mathrm{i}}$, la financiación incondicionada total correspondiente a la comunidad autónoma i que se ha determinado mediante la aplicación de las variables socioeconómicas.

$\mathrm{TC}_{\mathrm{i}}$, ingresos correspondientes a la comunidad autónoma i por tributos cedidos y tasas. $15 \% \mathrm{IRPF}_{\mathrm{i}}$, participación territorializada en el rendimiento del IRPF correspondiente a la comunidad autónoma i.

Este resultado, que evidentemente es cada vez menor en la medida en que se van incorporando nuevos mecanismos al modelo, se divide entre los ingresos tributarios no cedidos por el Estado, en concreto, los Ingresos Tributarios Ajustados Estructuralmente (ITAE), de tal forma que se obtiene ya el porcentaje de participación.

El nuevo modelo aprobado mantiene esta metodología, pero se producen cambios tanto en los ingresos tributarios como en las variables socioeconómicas que se van a aplicar para determinar los recursos correspondientes a cada comunidad autónoma. 
La autonomía del nuevo modelo

Como ya se ha indicado dicha autonomía viene dada por los ingresos de carácter tributario, que para el modelo que finaliza y el nuevo son los que se recogen en el siguiente cuadro.

\section{CUADRO 1}

\section{Comparación de los ingresos tributarios en el nuevo modelo}

\begin{tabular}{|l|l|}
\hline \multicolumn{1}{|c|}{ MODELO 1997-2001 } & \multicolumn{1}{c|}{ MODELO 2002-... } \\
\hline - Patrimonio & - Patrimonio \\
- Sucesiones y donaciones & - Sucesiones y donaciones \\
- Transmisiones patrimoniales & - Transmisiones patrimoniales \\
- Actos jurídicos documentados & - Actos jurídicos documentados \\
- Tasa sobre el juego & - Tasas sobre el juego \\
- 30\% IRPF & - 33\% IRPF \\
& - 35\% IVA \\
& - 40\% impuestos especiales sobre \\
& hidrocarburos, labores del taba- \\
& co, alcoholes y cerveza \\
& - 100\% impuesto especial sobre la \\
& electricidad y matriculación deve- \\
& hículos \\
\hline
\end{tabular}

Fuente: Elaboración propia.

Centraremos esta exposición en la idoneidad de la cesión del rendimiento de las principales figuras de nuestro sistema tributario, el IRPF y los impuestos sobre el consumo (IVA e impuestos especiales).

En todo este análisis conviene tener presente la siguiente idea. Desde el punto de vista de la eficiencia económica (objetivo último de la autonomía financiera), los ingresos tributarios que se deben asignar a cada comunidad autónoma, deben ser aquellos que se han generado en su propio territorio ${ }^{4}$.

4 Sobre esta cuestión cabe plantearse si dichos ingresos deben ser generados sólo por los residentes de la comunidad autónoma (población de derecho), o por la población de hecho. En el caso del IRPF los ingresos se generan por los residentes, en cambio los impuestos sobre 
A simple vista, puede parecer que el avance en autonomía del sistema es muy notable, de hecho se avanza en autonomía, pero el progreso real es menor que el aparente. La razón es que no existe capacidad normativa, ni de gestión, en muchos de estos tributos, además en el caso del IVA y de algunos impuestos especiales, no se cede parcialmente a las CCAA el tributo (como es el caso del IVA), sino que lo que se establece son participaciones territorializadas; en esta figura la autonomía es mínima, en realidad, las participaciones territorializadas tienen el carácter de una transferencia estatal. Analicemos detenidamente estas cuestiones.

Previamente hemos de tener en cuenta que la autonomía financiera es una cuestión de grado, es decir, en general todos los recursos de carácter incondicionado aportan autonomía al sistema, pero no en el mismo grado. Los instrumentos que tienen dicho carácter son: los tributos propios, los recargos sobre los tributos del Estado, los tributos cedidos total o parcialmente por el Estado, el endeudamiento, y las participaciones territorializadas en los tributos del Estado.

Para medir el grado de autonomía de estos recursos tenemos que analizar para cada uno de ellos las siguientes cuestiones ${ }^{5}$ : ¿a qué nivel de la Administración corresponde su configuración y reglamentación (capacidad normativa)?, ¿a qué nivel de la Administración correspondesu gestión?, y ¿quénivel se apropia del rendimiento del tributo? El grado de autonomía máximo se alcanzaría cuando el nivel al que corresponde cada una de estas tres cuestiones es el autonómico, es el caso de los tributos propios, los recargos y el endeudamiento. A continuación estarían los tributos cedidos, cuya gestión y rendimiento corresponde a las CCAA, pero la capacidad normativa es compartida con la Administración central. Menos autonomía que los tributos cedidos aporta la cesión parcial del IRPF, pues en este caso, tanto la capacidad normativa como el rendimiento son compartidos, y la gestión corresponde a la Administración central. En último lugar estarían las participaciones en impuestos, en las que la gestión y la capacidad normativa corresponden a la Administración central, y el rendimiento es compartido.

Que el IRPF es un tributo idóneo para formar parte del sistema de

el consumo se pagan por todas aquellas personas que a lo largo de un ejercicio han efectuado un consumo en la región, sean o no residentes de la misma.

5 Estas cuestiones son de carácter tributario; no obstante, las aplicaremos también al endeudamiento. 
financiación autonómica, es algo que ya ha puesto de manifiesto la experiencia autonómica de los últimos años. Ciertamente es un tributo del que se derivan diferencias financieras entre las CCAA, pero eso no quiere decir que el sistema sea insolidario, siempre y cuando se establezcan las medidas oportunas (transferencias) para compensar estas diferencias.

En el caso de los impuestos sobre el consumo (IVA, e impuestos especiales), las dificultades técnicas para descentralizarlos son mayores que en el IRPF. La principal dificultad para ceder a las CCAA el rendimiento de los impuestos sobre el consumo, es que éstos gravan la fase de fabricación (en el caso de los impuestos especiales, salvo el impuesto sobre matriculación de vehículos), o las distintas fases de transmisión del bien (en el caso del IVA), por lo que la comunidad en la que se recauda el tributo, y que por tanto percibiría el ingreso, puede no coincidir con la comunidad en que residen los sujetos pasivos que pagan dichos impuestos, es decir, el coste de la financiación de los servicios prestados por una comunidad autónoma puede recaer sobre los residentes de otras regiones. Esta es la razón por la que hasta ahora no se había planteado su incorporación al sistema.

Dichas dificultades técnicas siguen existiendo y, sin embargo, estos impuestos van a formar parte del nuevo sistema. ¿Qué razones han condicionado la nueva situación? Desde nuestro punto de vista, son las siguientes: por una parte, la necesidad casi indiscutible de aumentar la autonomía del sistema y el escaso margen para crear nuevos tributos propios por parte de las CCAA han determinado que el debate se centre en las figuras tributarias que aún estaban en manos de la Administración central; por otra parte, la concertación de éstos impuestos con las comunidades forales es un argumento para justificar que las dificultades técnicas no son insalvables, y que en la mayoría de las ocasiones las decisiones dependen de la voluntad política.

Según todo lo anterior, ¿qué valoración podemos hacer de la autonomía del nuevo modelo? Respecto al IRPF hay que indicar que en las primeras negociaciones se llegó a hablar de la posible cesión de un $40 \%$ de la tarifa del IRPF; el porcentaje finalmente acordado (el 33\%) nos parece más adecuado, sobre todo si tenemos en cuenta que es el principal instrumento de la política fiscal, la única política económica que ha quedado en manos del gobiernos centrales nacionales en la Unión Monetaria Europea. Por tanto, desde el punto de vista económico, éste es un tributo, que debe corresponder mayoritariamente a la administración central, aunque desde el punto de vista 
técnico es el más idóneo para ser descentralizado.

En el caso del impuesto sobre matriculación, al ser un tributo que grava la fase minorista, no existen razones técnicas que impidan su descentralización; además se conoce fácilmente la recaudación correspondiente a cada comunidad autónoma. Por el contrario, para asignar el IVA a cada comunidad se va a tener en cuenta un indicador del consumo relativo regional y, en el caso de los impuestos especiales, cabe esperar queel procedimiento empleado sea el mismo; ya hemos comentado que este tipo de recursos (participaciones territorializadas) apenas contribuyen a la autonomía, pues tienen el carácter de transferencia.

Respecto a la capacidad normativa, es uno de los puntos que están pendientes de ser definidos. En el caso del IRPF la capacidad normativa se refería a la posibilidad de modificar la tarifa autonómica del impuesto en un $20 \%$, y a establecer deducciones por circunstancias personales y familiares, pero no empresariales. El gobierno quiere eliminar este límite del $20 \%$, siempre y cuando las CCAA respeten la progresividad del tributo. La Unión Europea ha desaconsejado la cesión de capacidad normativa sobre el IVA, y de hecho no se va a ceder, y respecto al resto de tributos se plantea ceder capacidad normativa sobre los impuestos de matriculación y electricidad. La gestión seguirá en manos de la administración central.

Si tenemos en cuenta que las participaciones territorializadas en los impuestos indirectos, tienen el carácter de una transferencia, cabe preguntarse, ¿qué aportan estos nuevos recursos frente a la actual participación en los ingresos del Estado? Tanto la PIE como las participaciones territorializadas en los impuestos indirectos tienen el carácter de transferencias estatales, pero, mientras que la PIE crecería al ritmo en que lo hiciera el PIB (o según otro indicador acordado), los recursos tributarios crecerían según la recaudación efectiva de los mismos, de lo que cabe deducir que, los nuevos recursos no aportan más autonomía pero sí más dinero a las CCAA, que en definitiva es lo que van buscando.

La nivelación (solidaridad) en el nuevo modelo

Son pocas las referencias que tenemos sobre los cambios que el nuevo modelo va a introducir en la solidaridad del sistema. Por una parte, sabemos que se van a modificar las variables y ponderaciones, según las cuales se determina la financiación correspondiente a cada comunidad; por otra parte, 
aquellas CCAA que no lleguen a cubrir dicho volumen de recursos con los ingresos de carácter tributario, percibirán transferencias de la administración central hasta alcanzar los ingresos totales que les correspondan según la aplicación de las variables socioeconómicas. Analicemos, por tanto, los cambios de estas variables, los cuales se pueden observar en el siguiente cuadro.

CUADRO 2

Ponderaciones aplicadas a las variables de la PIE

\begin{tabular}{|l|l|c|c|c|c|c|}
\hline \multirow{4}{*}{} & \multirow{2}{*}{ VARIABLES } & \multicolumn{2}{|c|}{ CCAA 151 } & \multicolumn{2}{c|}{ CCAA 143 } & $\begin{array}{c}\text { NUEV0 } \\
\text { MODELO }\end{array}$ \\
\cline { 3 - 7 } & & $87-91$ & $92-96$ & $87-91$ & $92-96$ & $2002-\ldots$ \\
\hline \multirow{5}{*}{ Fondo General } & Población & 84,40 & 94,00 & 59,00 & 64,00 & 94,00 \\
\cline { 2 - 7 } & Superficie & 15,00 & 3,50 & 16,00 & 16,60 & 4,2 \\
\cline { 2 - 7 } & Dispersión & - & 0,60 & - & 2,00 & 1,2 \\
\cline { 2 - 7 } & Insularidad & 3,10 & 1,50 & 0,70 & 0,40 & 0,6 \\
\cline { 2 - 7 } & $\begin{array}{l}\text { Unidades } \\
\text { administrativas }\end{array}$ & - & 0,40 & 24,30 & 17,00 & - \\
\hline \multirow{2}{*}{$\begin{array}{l}\text { Fondo Redistributivo } \\
\text { (suma cero) }\end{array}$} & Pobreza relativa & 1,99 & 2,70 & 4,20 & 2,70 & - \\
\cline { 2 - 8 } & Esfuerzo fiscal & 3,08 & 1,82 & 5,00 & 1,82 & - \\
\hline
\end{tabular}

Fuente: Elaboración propia

En el modelo que ha estado vigente desde 1997 hasta 2001, las variables y ponderaciones aplicadas fueron las mismas que las del modelo aplicado en el quinquenio 1992-1996.

Como se desprende del cuadro anterior, hasta el año 2001 se distinguía entre dos grupos de CCAA, las que se conocen como comunidades del artículo 151 de la Constitución, y las del 143; esta distinción, como ya se ha visto, se deriva del distinto nivel de competencias de las comunidades. Dado que a lo largo del último quinquenio ya se han transferido las competencias en educación a todas las CCAA, en el próximo modelo se elimina esta

\section{RFS}


distinción y se aplican las mismas variables y ponderaciones a todas las comunidades.

Por otra parte, hay un grupo de variables que constituyen un fondo general; respecto a este grupo en el nuevo modelo desaparece la variable unidades administrativas. Desde nuestro punto de vista, la desaparición de dicha variable es acertada, ya que perjudicaba a las comunidades uniprovinciales $^{6}$. El peso de la variable población, en el nuevo modelo, coincide con el peso que ya tenía esta variable en el grupo de comunidades del 151.

El segundo fondo, de carácter redistributivo, estaba compuesto por dos variables, la pobreza relativa y el esfuerzo fiscal; este último se estimaba según el IRPF. Este fondo sufrió modificaciones a raíz de la participación de las CCAA en el IRPF. En el nuevo modelo, está previsto un fondo de renta relativa, es decir, se elimina la variable esfuerzo fiscal, y se realiza una redistribución de recursos entre CCAA únicamente en función de su renta relativa.

Otra cuestión importante es la que se refiere al año de referencia que se toma para los datos. En 1997 no se actualizó y, por ejemplo, el censo de población que se empleaba era el correspondiente a 1988. En el nuevo modelo está prevista la actualización de la variable población con el censo de 1999. Esta cuestión ha sido el principal campo de batalla de la comunidad autónoma andaluza, desde 1997, y aún hoy está pendiente de llegar a un acuerdo con la administración central, sobre una posible compensación por los recursos que Andalucía ha dejado de ingresar debido a la no actualización, en 1997, de esta variable. Esta reclamación es lícita, pues no había razones económicas, ni financieras que justificaran la no actualización del censo de población; fue una decisión política.

6 El índice de unidades administrativas se calculaba asignando el valor 0,5 a cada comunidad autónoma y a cada provincia, así por ejemplo, a Cataluña le correspondía el valor 2,5 que se obtenía al sumar 0,5 por cada provincia y 0,5 por los servicios centrales de la comunidad. Lógicamente, las comunidades uniprovinciales recibían menos recursos por la aplicación de esta variable.

7 La razón que argumentó el gobierno central, es quela actualización de las variables sellevaría a cabo cuando se transfiera a todas las CCAA las competencias en educación. En realidad, de esta forma el gobierno se ahorró los mayores recursos que tendría que haber transferido a las CCAA cuya población había incrementado sensiblemente, además de evitarse el enfrentamiento con las comunidades que perdían población, entre las que se encontraba a la cabeza, Cataluña, artífice, por cierto, del modelo aprobado en 1997. 
Desde nuestro punto de vista, la nueva configuración de las variables es más acertada que la que existía hasta ahora. Sin embargo, hay que objetar que tanto las variables como sus ponderaciones son fruto del consenso político, y no se derivan de un estudio económico del gasto regional, por lo que cabría cuestionar hasta qué punto realmente se compensa a las CCAA por el distinto coste de prestación de los servicios.

Por último, en el nuevo modelo se recogen otros recursos de carácter condicionado; por ejemplo, está previsto establecer asignaciones (transferencias) de nivelación, para financiar los costes de la educación y la sanidad.

\section{Conclusiones}

La descentralización del sector público tiene una justificación económica: realizar una asignación del gasto más eficiente. Este objetivo de asignación eficiente del gasto público, está directamente relacionado con uno de los principios de la financiación autonómica, la autonomía financiera. Pero además, la autonomía financiera es necesaria para que los gobiernos regionales dispongan de una efectiva autonomía política, y para evitar situaciones de irresponsabilidad por parte de dichos go biernos. Además del principio de autonomía, el sistema de financiación de las CCAA debe hacer efectivos los principios de solidaridad, coordinación y suficiencia.

El grado en que se alcancen los objetivos de autonomía y solidaridad, depende de los tipos de ingresos con que se financie el gasto regional, es decir, no todos los recursos financieros contribuyen a alcanzar la autonomía, o la solidaridad en la misma medida. Así por ejemplo, para el logro de los objetivos propios de la autonomía, los ingresos tributarios son preferibles a las transferencias, pero, a su vez, dentro de los primeros, la cesión de un tributo a los gobiernos regionales es preferible al establecimiento de participaciones territorializadas. Las transferencias del Estado no contribuyen a la autonomía, pero son el principal mecanismo financiero para hacer efectivos los objetivos propios del principio de solidaridad.

Al valorar el nuevo modelo de financiación autonómica, hemos detener en cuenta, no sólo el volumen total de recursos que va a percibir cada comunidad autónoma, sino también el origen de dichos ingresos, porque esta última cuestión es la que afecta al logro de los objetivos propios de la autonomía y de la solidaridad. 
Respecto a la primera cuestión, la autonomía, el margen para avanzar en la autonomía del sistema de financiación por la vía de la cesión de los impuestos estatales, no era muy amplio, y cualquier acuerdo que se adoptara en esta línea estaría técnicamente forzado. De hecho, para salvar los inconvenientes técnicos que se presentan en la cesión del rendimiento de los impuestos sobre el consumo, por ejemplo, el IVA, se ha optado por el establecimiento de participaciones territorializadas, es decir, repartir estos ingresos, en función del consumo relativo regional. Como se ha expuesto, esta solución apenas contribuye a la autonomía del sistema, pero, ciertamente, va a aportar más ingresos a las CCAA. En el caso de los impuestos especiales, una solución más satisfactoria hubiera sido cambiar la configuración de los impuestos que gravan la fase de fabricación y transformarlos en impuestos que graven la fase minorista. Tanto la LOFCA como la Ley de Cesión de Tributos del Estado a las CCAA fueron reformadas en 1996, para dar cabida a la cesión parcial del IRPF en el sistema, así como a la cesión de capacidad normativa sobre los tributos estatales cedidos a las CCAA. Sin embargo, pocas veces se ha planteado cambiar la configuración de los impuestos especiales para darles cabida en la LOFCA ${ }^{8}$.

También hay que recordar que la gestión y la capacidad normativa siguen en manos de la administración central, por lo que a modo de conclusión cabe afirmar que el modelo avanza en autonomía, pero no tanto como aparenta. El sistema debía avanzar en dotar de mayor autonomía a las CCAA, pero la solución a esta última cuestión no estaba tanto en la cesión de los impuestos estatales, sino en la creación de nuevos tributos propios de las CCAA, en el establecimiento de recargos sobre los tributos del Estado, o en el ejercicio de la capacidad normativa cedida.

Por otra parte, la nivelación de recursos se concreta en reducir las diferencias financieras entre CCAA. Cómo medir dichas diferencias financieras es una cuestión técnica, que no tiene una única solución; cuánto se deben reducir estas diferencias, es una decisión política. Sin duda, las variables socioeconómicas que se emplean para determinar los ingresos de las CCAA, guardan relación con el coste de prestación de los servicios, pero no son las únicas: son todas las que están, pero no están todas las que son. Cuanto más queramos aproximarnos a una estimación real del coste de prestación de los

8 La LOFCA recoge entre los tributos susceptibles de cesión a las CCAA los impuestos sobre consumos específicos que recaigan sobre la fase minorista. 
servicios públicos, mayor número de variables habrá que contemplar, y más complejo será el sistema. Entre esta situación (contemplar todas las variables), y la actual (en que se contemplan cuatro variables), existe otra alternativa: emplear como única variable la población. Son varias las razones que cabe argumentar para ello, pero, sin duda, la principal es que el coste de prestación de los servicios depende fundamentalmente de la población. Si la población fuera la única variable a tener en cuenta, entonces el reparto de la financiación sería tal que los ingresos per cápita serían los mismos en cada región; no habría, por tanto, diferencias financieras por el lado de los ingresos. No obstante, varios go biernos autonómicos no estaban dispuestos a renunciar a otro tipo de variables; en cualquier caso, hay que aceptar que cualquier decisión que se tome en este sentido no va a satisfacer por igual a todas las regiones, porque sus realidades socioeconómicas son distintas.

Finalmente, hay que valorar positivamente que el modelo acordado ha sido consensuado entre todas las CCAA y que, por tanto, a diferencia del anterior modelo, ninguna comunidad va a quedar al margen del mismo; eso quiere decir, que, en líneas generales, todas las CCAA consideran que es un buen modelo. 


\section{Bibliografía}

Adame Martínez, F. (1998), El sistema de financiación de las CCAA de régimen general, Ed. Comares, Granada, pp. 326.

Agúndez Garcia, A. y Pedreja Chaparro, F. (1991), "Nivelación de servicios en haciendas descentralizadas: comentarios al libro blanco", Papeles de Economía Española, núm. 69, pp. 192-199.

Biescas Ferrer, J.A. (1999), "Autonomía, responsabilidad y nivelación en la financiación autonómica", Rev. de Economía Aragonesa, Ponencias de la XXIV Reunión de Estudios Regionales, pp. 19.

CalsamiglaA, X. (1990), "La financiación de las Comunidades Autónomas y el principio de solidaridad", Rev. de Economía Pública, núm. 6, pp. 3-45.

Calsamigla, X. (1991), “Criterios distributivos para un mecanismo solidario de financiación de las Comunidades Autónomas", Presupuesto y Gasto Público, núm. 5, pp. 93-104.

Castells Oliveres, A. (2000), "Autonomía y solidaridad en el sistema de financiación autonómica", Papeles de Economía Española, núm. 83, pp. 37-59.

Castells Oliveres, A. y Solé Ollé, A. (2000), Cuantificación de las necesidades de gasto de las Comunidades Autónomas: metodología y aplicación práctica, Instituto de Estudios Fiscales, Madrid, pp. 550.

Ezquiaga Domínguez, I. y García de Bustos, F. (1997), “Una evaluación del sistema de financiación autonómica para el quinquenio 1997-2001", Cuadernos de Información Económica, núm. 120-121, pp. 173-192.

Herrera Maldonado, J.M. y Hierro Recio, L.A. (1997), “El componente de solidaridad en la financiación de las Comunidades Autónomas", I Congreso de Ciencia Regional de Andalucía, Jerez, abril, pp. 34-40.

Monasterio Escudero, C. (2000a), “La financiación subcentral en España. Principios y desarrollo", Papeles de Economía Española, núm. 83, pp. 25-36.

Ríos BerJ ILLOS, A. DE LOS, (2001), Una propuesta de financiación para las Comunidades Autónomas españolas de régimen común. Un enfoque económico. Tesis Doctoral.

Sánchez Maldonado, J. y Gómez Sala, J. (1996a), "El impuesto sobre el valor añadido como tributo descentralizado: análisis del caso español", Rev. de Estudios Regionales, núm. 45, pp. 43-88. 
Sánchez Maldonado, J. y Gómez Sala, J. (1996b), "La imposición sobre el consumo y la financiación autonómica", Papeles de Economía Española, núm. 69, pp. 212-226.

Tanco Martín-Criado, J. y Duro Álvarez, P. (1997), "Corresponsabilidad Fiscal y Solidaridad Interterritorial", I Congreso de Ciencia Regional de Andalucía, Jerez, abril, pp. 1-25. 